\title{
DDI-RDF Discovery - A Discovery Model for Microdata
}

\author{
by Thomas Bosch', Olof Olsson², Benjamin Zapilko³, Arofan \\ Gregory ${ }^{4}$, and Joachim Wackerow ${ }^{5}$
}

\begin{abstract}
Ontology engineers and experts from the social, behavioral, and economic sciences developed a data discovery ontology covering a subset of both the DDI Codebook and Lifecycle models, and implemented a rendering of DDI XML instances to RDF (Resource Description Framework). The main goals associated with the design process of the DDI ontology were to reuse widely adopted and accepted ontologies like Dublin Core (DC) and Simple Knowledge Organization System (SKOS) and also to define meaningful relationships to the RDF Data Cube vocabulary. Now, organizations have the possibility to publish their DDI data and metadata in RDF and link it with many other datasets from the Linked Open Data (LOD) cloud. As a consequence, a huge number of related DDI instances can be discovered, queried, connected, and harmonized. The combination of DDI metadata (as well as data) from several organizations, based on this RDF discovery (Disco) vocabulary, will enable powerful derivations of implicit knowledge out of explicitly stated pieces of information.
\end{abstract}

Keywords: Semantic

Web, Linked Data,

Ontology Design, DDI

\section{Data \\ Documentation \\ Initiative: \\ Background}

\section{Overview}

The DDI specification describes social science data, data covering human activity, and other data based on observational methods measuring real-life phenomena. DDI supports the entire research data lifecycle. DDI metadata accompany and enable data conceptualization, collection, processing, distribution, discovery, analysis, repurposing, and archiving. Metadata is structured information that describes, explains, locates, or otherwise makes it easier to retrieve, use, or manage data (NISO Press,
2004). DDI does not invent a new model for statistical data. It formalizes state of the art concepts and common practice in this domain. DDI focuses on both microdata and aggregated data. It has its strength in microdata -- data on the characteristics of units of a population, such as individuals or households, collected by, for example, a census or a survey. Statistical microdata are not to be confused with microdata in HTML, an approach to nest semantics within web pages. Aggregated data (e.g., multidimensional tables) are likewise covered by DDI. They provide summarized versions of the microdata in the form of statistics like means or frequencies. Publicly accessible metadata of good quality are important for finding the right data. This is especially the case if access to microdata is restricted due to potential risk of disclosure of respondent identities. DDI is currently specified in XML Schema, organized in multiple modules corresponding to the individual stages of the data lifecycle, and includes over 800 elements (DDI Lifecycle).

A specific DDI module (using the simple Dublin Core namespace) allows for the capture and expression of native Dublin Core elements, used either as references

\section{DDI has its strength in the domain of social, economic, and behavioral data}

or as descriptions of a particular set of metadata. This is used for citation of the data, parts of the data documentation, and external material in addition to the richer, native DDI. This approach supports applications that understand the Dublin Core XML, but do not understand DDI. DDI is aligned with other metadata standards as well, with SDMX ${ }^{6}$ (time-series data) for exchanging aggregate data, ISO/IEC 11179 (metadata registry) for building data registries such as question, variable, and concept banks (ISO/IEC, 


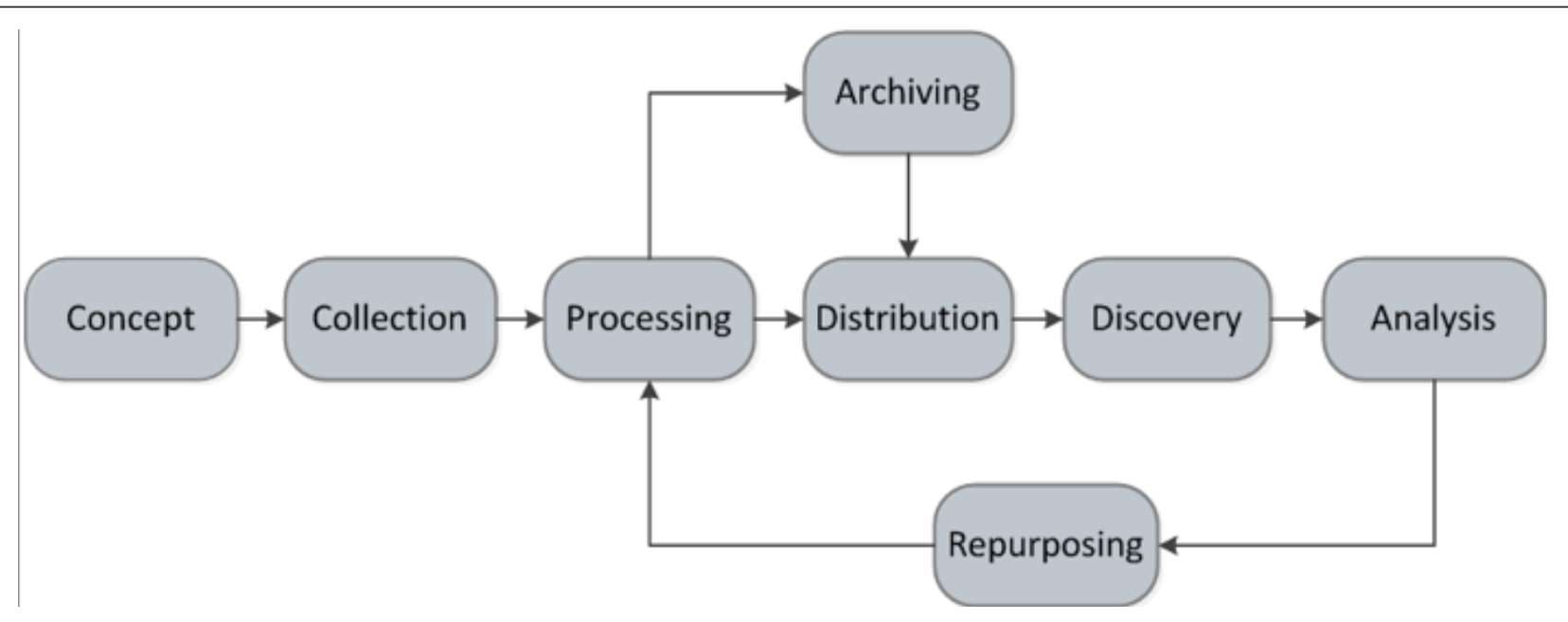

Figure 1. DDI Data Lifecycle

2004), and ISO 19115 (geographic standard) for supporting GIS (geographic information system) users (ISO 19115-1:2003, 2003).

\section{Goals}

DDI supports technological and semantic interoperability in enabling and promoting international and interdisciplinary access to and use of research data. Structured metadata with high quality enable secondary analysis without the need to contact the primary researcher who collected the data. Comprehensive metadata (potentially along the whole data lifecycle) are crucial for the replication of analysis results in order to enhance research transparency. DDI also enables the reuse of metadata of existing studies (e.g., questions, variables) for designing new studies, an important ability for repeated surveys and for comparison purposes. DDI supports researchers who follow the above mentioned goals.

\section{DDI Users}

A large community of data professionals, including data producers (e.g., of large, academic international surveys), data archivists, data managers in national statistical agencies and other official data producing agencies, and international organizations use the DDI metadata standard. The DDI Alliance hosts a comprehensive list of projects using the DDI ${ }^{7}$. Academic users include the UK Data Archive at the University of Essex ${ }^{8}$, the Dataverse Network at the Harvard-MIT Data Center ${ }^{9}$, and the Inter-university Consortium for Political and Social Research (ICPSR) at the University of Michigan ${ }^{10}$. Official data producers in more than 50 countries include the Australian Bureau of Statistics (ABS) ${ }^{11}$ and many national statistical institutes of the Accelerated Data Program for developing countries $^{12}$. Examples of international organizations using DDI are UNICEF, the Multiple Indicator Cluster Surveys (MICS) ${ }^{13}$, The World Bank ${ }^{14}$, and The Global Fund to Fight AIDS, Tuberculosis and Malaria ${ }^{15}$

\section{DDI History and Versions}

The DDI project, which started in 1995, has steadily gained momentum and evolved to meet the needs of the social science research community. In 2003, the DDI Alliance was established to develop and promote the DDI specification and associated tools, education, and outreach program. The DDI Alliance is a selfsustaining membership organization whose institutional members have a voice in the development of the DDI specification. To ensure continued support and ongoing development of the standard, DDI has been branched into two separate development lines. DDI-Codebook (formerly DDI2) is a more light-weight version of the standard, intended primarily to document simple survey data for archival purposes. Encompassing all of the DDI-Codebook specification and extending it, DDI-Lifecycle (formerly DDI3, first version published in 2008) is designed to document and manage data across the entire data lifecycle, from conceptualization to data publication and analysis and beyond.

\section{Data Lifecycle}

The common understanding is that both statistical data and metadata are part of a data lifecycle (Figure 1 displays this lifecycle -- it is described in more detail on the DDI Alliance website $\left.{ }^{16}\right)$. Multiple institutions are involved in the data lifecycle, which is an interactive process with multiple feedback loops. Data documentation is a process, not an end condition where a final status of the data is documented. Rather, metadata production should begin early in a project, and metadata should continue to be captured at the source as data come into being. The metadata can then ideally be reused along the data lifecycle. Such practice would incorporate documentation as part of the research method (Jacobs et al., 2004). A paradigm change would be enabled: on the basis of the metadata, it becomes possible to drive processes and generate items like questionnaires, statistical command files, and web documentation, if metadata creation is started at the design stage of a study (e.g., survey) in a well-defined and structured way.

\section{Limitations}

DDI has its strength in the domain of social, economic, and behavioral data. Ongoing work focuses on the early phases of survey design and data collection as well as on other data sources like register data. The next major version of DDI will incorporate the results of this work. It will be opened to other data sources and to data of other disciplines.

\section{Related Work}

With respect to documenting data, there are several relevant metadata standards like SDMX (Statistical Data and Metadata Exchange) for the representation and exchange of aggregated data, ISO 19115 (ISO 19115-1:2003, 2003) for geographic information, and PREMIS ${ }^{17}$ for preservation purposes. The metadata registry standard ISO 11179 (ISO/IEC, 2004) addresses the modeling 


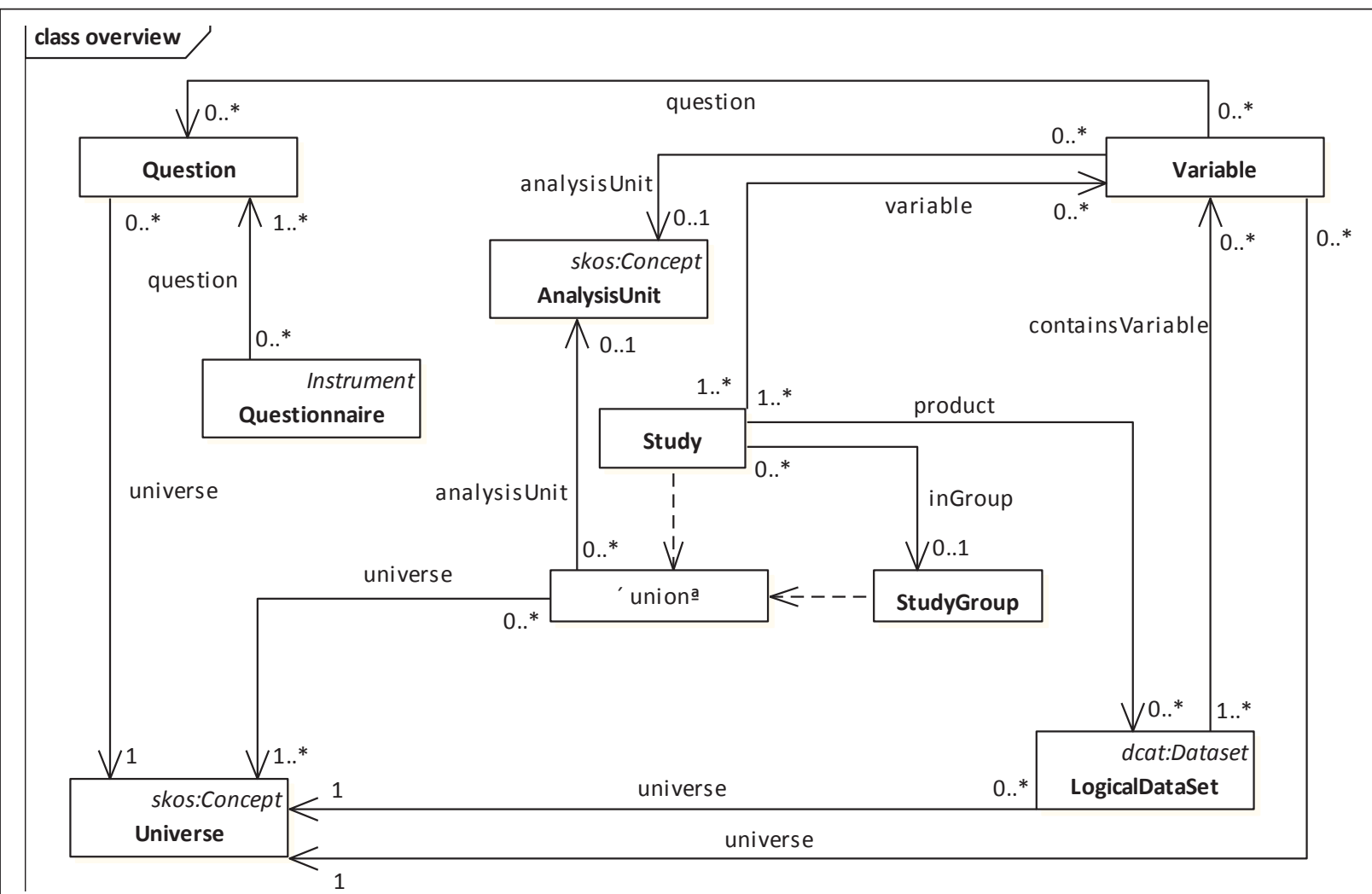

Figure 2. DDI-RDF Discovery Vocabulary (Disco)

of metadata, e.g., reference models, and registries. However, there are as yet few adequate RDF-based vocabularies for documenting data. DDI-RDF for discovery, or Disco, has a clearly defined focus on describing microdata, which has not been covered to this extent by other established vocabularies yet. Therefore it fits well alongside other metadata standards on the web and can clearly be distinguished. Connection points to classes or properties of other vocabularies ensure equivalent or more detailed possibilities for describing entities or relationships.

An RDF expression of the Simple Dublin Core specification exists which could be used for citation purposes (DCMI, 2008). Furthermore, the DCMI Metadata Terms (DCMI, 2010) have been applied when suitable for representing basic information about publishing objects on the web as well as for hasPart relationships. For representing concepts that are organized in ways similar to thesauri and classification systems, classes and properties of Simple Knowledge Organization System (SKOS) ${ }^{18}$ have been used. Some aspects of DDI-RDF are already similarly represented in other metadata vocabularies, e.g., data management and documentation. The vocabulary of interlinked datasets (VoID) ${ }^{19}$ represents relationships between multiple datasets, while the Provenance Vocabulary ${ }^{20}$ provides the possibility to describe information on ownership and can be used to represent and exchange provenance information generated in different systems and under different contexts. In this context, a study can be seen as a data-producing process and a logical dataset as its output artifact. Data Catalog Vocabulary (DCAT) ${ }^{21}$ is an RDF vocabulary designed to facilitate interoperability between data catalogs published on the Web. By using DCAT to describe datasets in data catalogs, publishers increase discoverability and enable applications easily to consume metadata from multiple catalogs.
An established RDF metadata vocabulary, which seems similar to DDI-RDF at first glance, is the RDF Data Cube vocabulary (Cyganiak et al., 2010). This model maps the SDMX information model to an ontology and is therefore compatible with the cube model that underlies SDMX. It can be used for representing aggregated data (also known as macrodata) such as multidimensional tables. Aggregate data are data derived from microdata by statistics on groups or aggregates, such as counts, means, or frequencies. A dataset presented with the Data Cube vocabulary consists of a set of values organized along a group of dimensions, which is comparable to the representation of data in an Online Analytical Processing system. In the Data Cube vocabulary associated metadata are added.

\section{DDI as Linked Data}

Statistical domain experts (core members of the DDI Alliance Technical Implementation Committee, representatives of national statistical institutes, national data archives) and Linked Open Data community members have chosen the DDI elements that are seen as most important to solve problems associated with diverse identified use cases around data discovery. Widely accepted and adopted vocabularies are reused to a large extent. There are features of DDI that can be addressed through other vocabularies, such as: describing metadata for citation purposes using Dublin Core, describing aggregated data like multidimensional tables using the RDF Data Cube Vocabulary ${ }^{22}$, and delineating code lists, category schemes, mappings between them, and concepts like topics using SKOS. This section serves as an overview of the conceptual model for the Disco vocabulary. More detailed descriptions of all the properties are given in the specification ${ }^{23}$ and a conference paper (Bosch et al. 2012). 


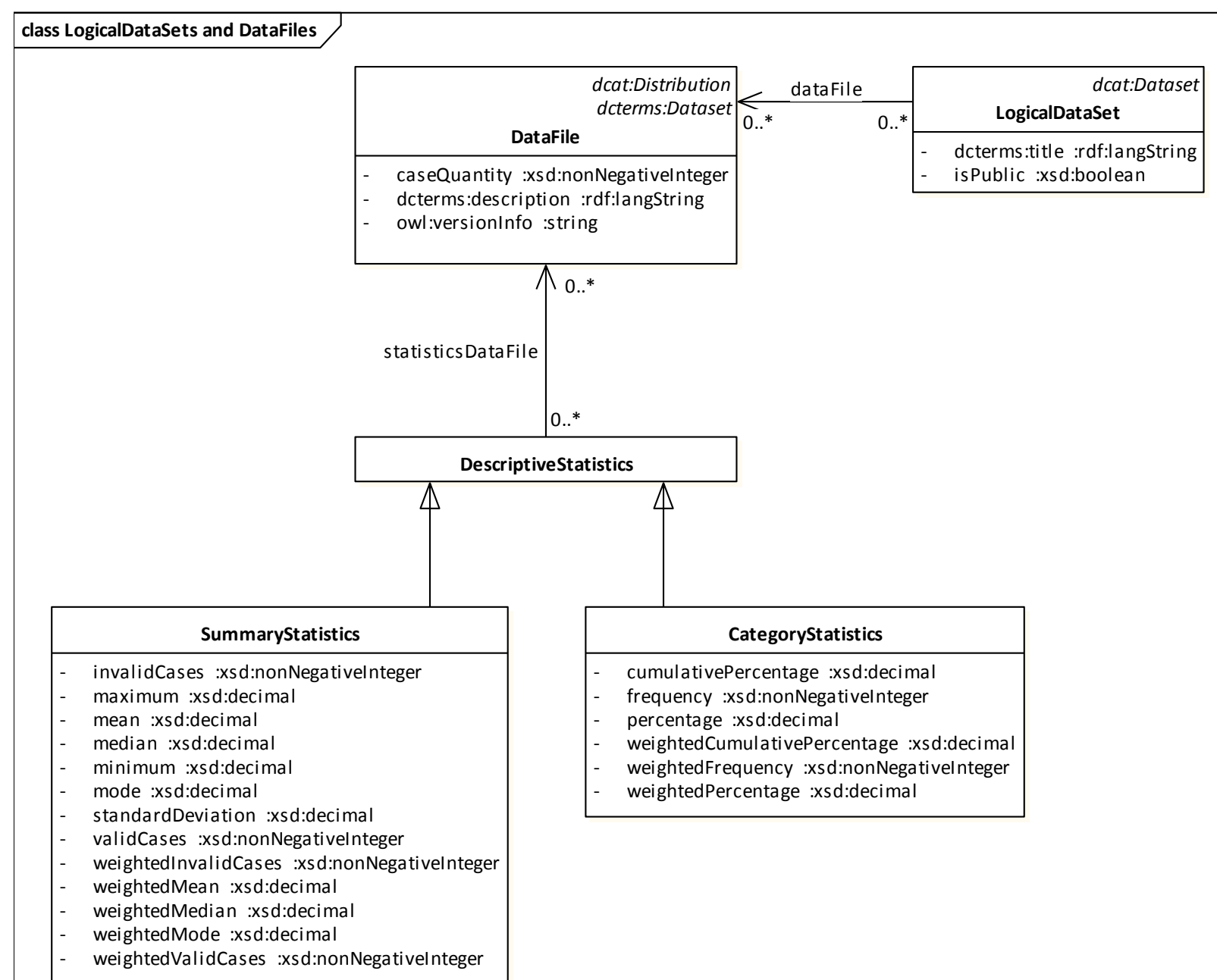

Figure 3. LogicalDataSets and DataFiles

\section{Overview}

Figure 2 provides a diagram of the conceptual model containing a small subset of the DDI-XML specification ${ }^{24}$. To understand the DDI Discovery Vocabulary, there are a few central classes, which can serve as entry points. The first of these is Study. A Study represents the process by which a dataset was generated or collected. Literal properties include information about the funding, organizational affiliation, abstract, title, version, and other such high-level information. In some cases, where data collection is cyclic or ongoing, datasets may be released as a StudyGroup, where each cycle or "wave" of the data collection activity produces one or more datasets. This is typical for longitudinal studies, panel studies, and other types of "series". In this case, a number of Study objects would be collected into a single StudyGroup.

Datasets have two representations: a logical representation, which describes the contents of the dataset, and a physical representation, which is a distributed file holding that data. It is possible to format data files in many different ways, even if the logical content is the same. LogicalDataSet represents the content of the file (it is organized into a set of Variables). The LogicalDataSet is an extension of the dcat:DataSet. Physical, distributed files are represented by the DataFile, which is itself an extension of dcat:Distribution.
When it comes to understanding the contents of the dataset, this is done using the Variable class. Variables provide a definition of the column in a rectangular data file, and can associate it with a Concept and a Question (the Question in the Questionnaire which was used to collect the data). Variables are related to a Representation of some form, which may be a set of codes and categories (a "codelist") or may be one of other normal data types (dateTime, numeric, textual, etc.). Codes and Categories are represented using SKOS concepts and concept schemes.

Data are collected about a specific phenomenon, typically involving some target population, and focusing on the analysis of a particular type of subject. These are respectively represented by the classes Universe and AnalysisUnit. If, for example, the adult population of Finland is being studied, the AnalysisUnit would be individuals or persons.

Unique identifiers for specific DDI versions are used for easing the linkage between DDI-RDF metadata and the original DDI-XML files. Every element can be related to any foaf:Document (DDI-XML files) using dcterms:relation. Any entity can have version information (owl:versionlnfo). However, the most typical cases are the versioning of the metadata (the DDI or the RDF file), the versioning of the study (as a study goes through the lifecycle from conception through data collection), and the versioning of the data files. Every LogicalDataSet may have access rights statements (dcterms:accessRights) and 
licensing information (dcterms:license) attached to it. Studies, logical datasets, and data files may have spatial (dcterms:spatial), temporal (dcterms:temporal), and topical (dcterms:subject) coverage.

\section{Studies and StudyGroups}

A simple Study supports the stages of the full data lifecycle in a modular manner. As noted above, a Study represents the process by which a dataset was generated or collected, and a number of Study objects can be collected into a single StudyGroup.

Studies may have multiple disco:instrument relationships to Instruments and may have disco:dataFile connections with 0 to $n$ DataFiles. Studies are associated with 0 to $n$ Variables using the object property disco:variable. Studies may have multiple LogicalDataSets (disco:product). Studies or StudyGroups (the union of Study and StudyGroup) may have an abstract (dcterms:abstract), a title (dcterms:title), a subtitle (disco:subtitle), an alternative title (dcterms:alternative), a purpose (disco:purpose), and information about the date and time the Study was made publicly available (dcterms:available). Disco:kindOfData describes the kind of data documented in the logical product(s) of a Study (e.g., survey data or administrative data). Disco:ddiFile leads to foaf:Documents which are the DDI-XML files containing further descriptions of the Study or the StudyGroup. Creators (dcterms:creator), contributors (dcterms:contributor), and publishers (dcterms:publisher) of Studies and StudyGroups are foaf:Agents which are either foaf:Persons or org:Organizations whose members are foaf:Persons. Studies and StudyGroups may be funded by (disco:fundedBy) foaf:Agents. The object property disco:fundedBy is defined as sub-property of dcterms:contributor

Universe is the total membership or population of a defined class of people, objects, or events. AnalysisUnit is the particular type of subject being analyzed, for example, individuals or persons. Studies and groups of Studies must have 1 to n Universes which are subclasses of skos:Concepts. For Universes one can state definitions using skos:definition. The union of Study and StudyGroup may have 0 or 1 AnalysisUnit reached by the object property disco:analysis Unit. AnalysisUnit is specified as a sub-class of skos:Concept.

Logical Datasets, Data Files, Descriptive Statistics, and Aggregated Data

As noted, datasets have a logical representation, which describes the contents of the dataset, and a physical representation, which is a distributed file holding that data. It is possible to format data files in many different ways, even if the logical content is the same. LogicalDataSet represents the content of the file (its organization into a set of Variables). The LogicalDataSet is an extension of dcat:DataSet. Physical, distributed files containing the microdata datasets are represented by DataFile, which are sub-classes of dcterms:Datasets and dcat:Distribution.

An overview of the microdata can be given either by descriptive statistics or aggregated data. DescriptiveStatistics may be minimal, maximal, mean values, and absolute and relative frequencies. qb:DataSet originates from the RDF Data Cube Vocabulary ${ }^{25}$, an approach to map the SDMX information model to an ontology. A DataSet represents aggregated data such as multidimensional tables. SummaryStatistics pointing to variables and CategoryStatistics pointing to categories and codes are both descriptive statistics.

\section{Variables, Variable Definitions, Representations, and Concepts}

When it comes to understanding the contents of the dataset, this is done using the Variable class. Variables provide a definition of the column in a rectangular data file, and can associate it with a Concept, and a Question. Variable is a characteristic of a unit being observed. A Variable might be the answer to a question, have an administrative source, or be derived from other Variables. VariableDefinitions encompass study-independent, reusable parts of Variables like occupation classification.

\section{Questions, Variables, and VariableDefinitions may have} Representations. Representation is defined as a sub-class of the union of rdfs:Datatype (e.g., numeric or textual values) and skos:ConceptScheme, as for example questions may have as their response domain a mixture of a numeric response domain containing numeric values (rdfs:Datatype) and a code response domain (skos:ConceptScheme) -- a set of codes and categories (a "codelist").

Codes and Categories are represented using SKOS Concepts and concept schemes. SKOS defines the term skos:Concept, which is a unit of knowledge created by a unique combination of

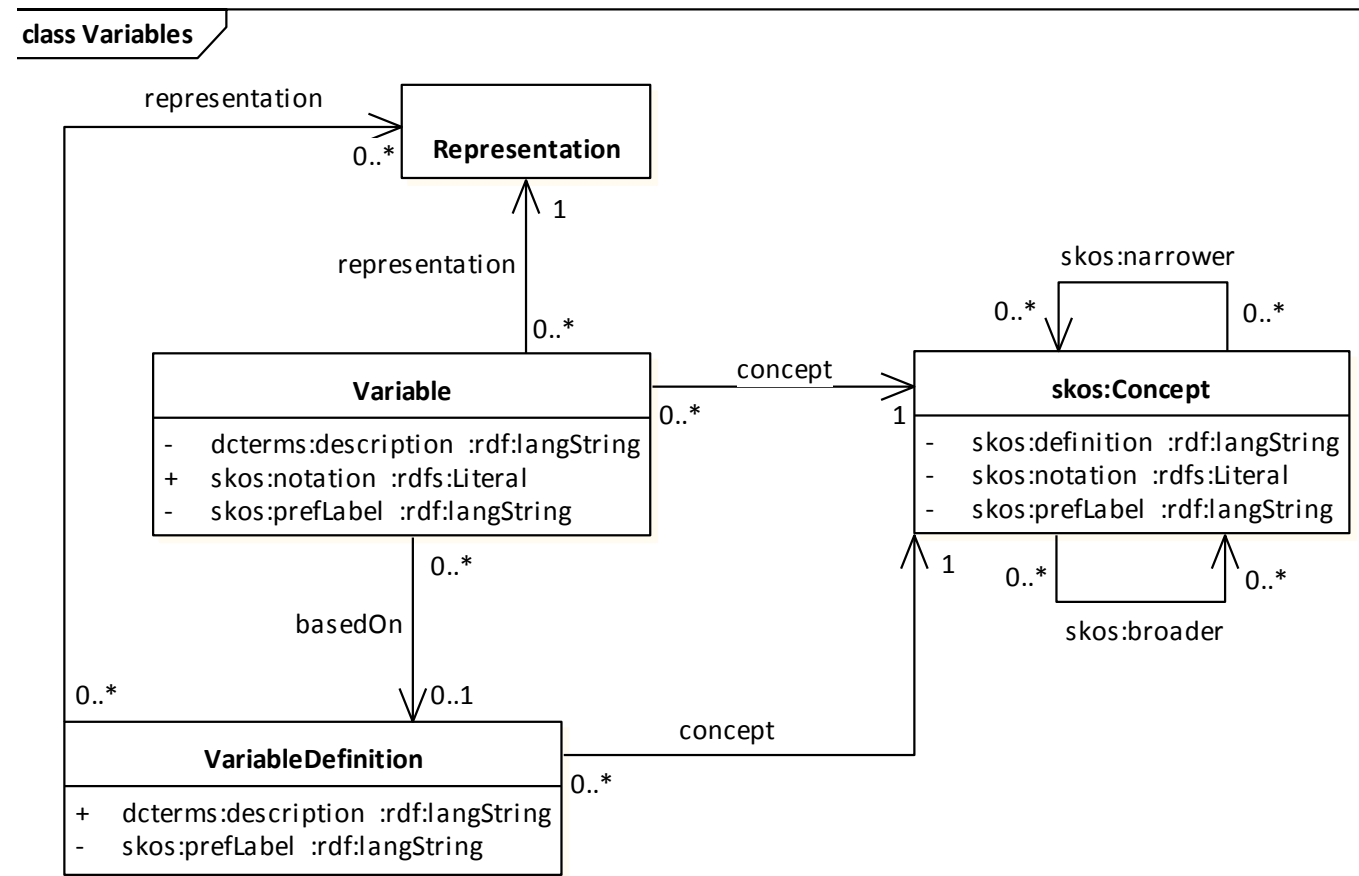

Figure 4. Variables 


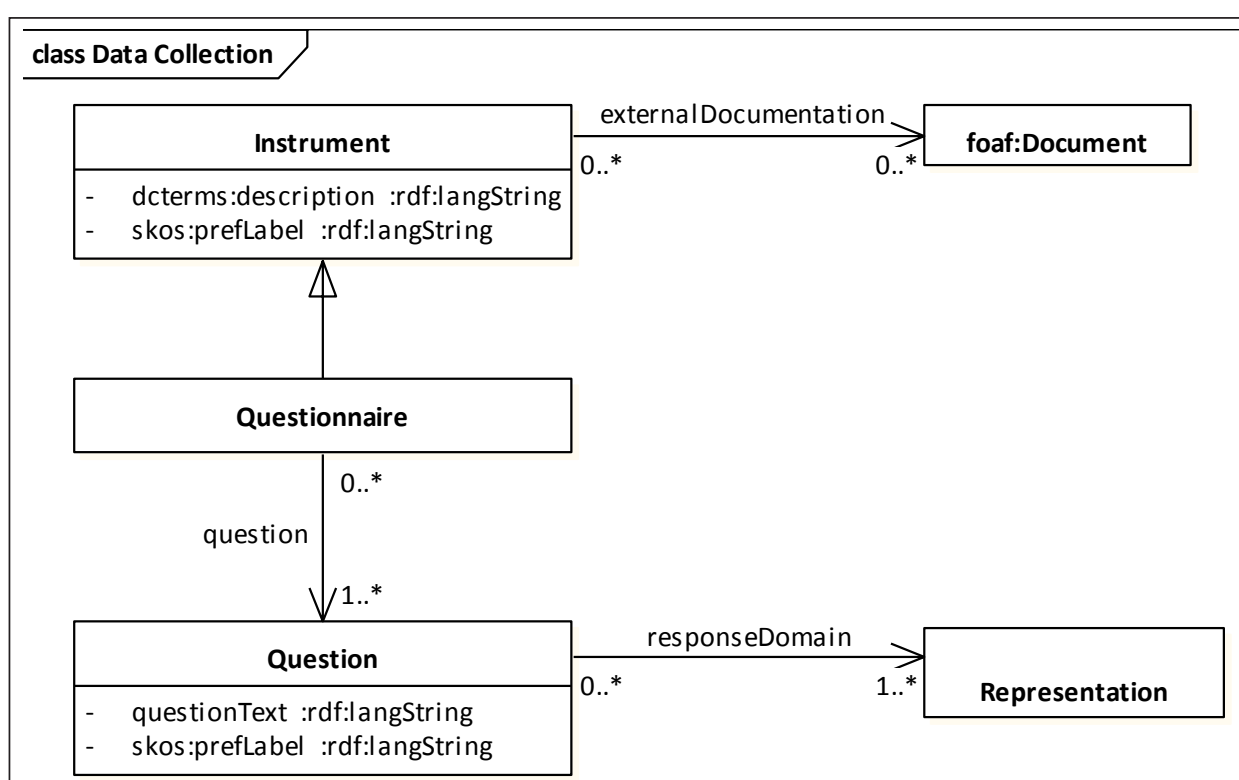

Figure 5. Data Collection
Enhancing Discovery of Data by Providing Related Metadata

Many archives and government organizations have large amounts of data, sometimes publicly available, but often confidential in nature, requiring applications for access. While the datasets may be available (typically as CSV files), the metadata which accompanies them is not necessarily coherent, making the discovery of these datasets difficult. A prospective user has to read related documents to determine if the data are useful for his/her research purposes. The data provider could enhance discovery of data by providing key metadata in a standardized form. This would allow the creation of standard queries to programmatically identify datasets. The DDI-RDF Discovery Vocabulary would support this approach. characteristics. In the context of statistical (meta)data, concepts are abstract summaries, general notions, or knowledge of a whole set of behaviors, attitudes, or characteristics which are seen as having something in common. Concepts may be associated with variables and questions. A skos:ConceptScheme is a set of metadata describing statistical concepts. Skos:Concept is reused to a large extent to represent DDI concepts, codes, and categories.

\section{Data Collection}

The data for the study are collected by an Instrument. The purpose of an Instrument, e.g., an interview, a questionnaire, or another entity used as a means of data collection, is in the case of a survey to record the flow of a questionnaire, its use of questions, and additional component parts. A Questionnaire contains a flow of questions. A Question is designed to elicit information on a subject, or sequence of subjects, from a respondent. The next figure visualizes the datatype and object properties of Instrument and Question.

One can describe (dcterms:description) Instruments and associate labels (skos:prefLabel) to Instruments. Instruments may have multiple external documentation files of the type foaf:Document. Questionnaires are special instruments having at least one collection mode (disco:collectionMode) which is a skos:Concept. Questionnaires must contain at least one Question. Questions have a question text (disco:questionText), a label (skos:prefLabel), exactly one universe (disco:universe), multiple concepts (disco:concept), and at least one response domain (disco:responseDomain).

\section{Use Cases}

This section describes the scenarios that the DDI-RDF Discovery Vocabulary was designed to support. These are not formal UML use cases -- instead, they are scenarios for the possible use of the vocabulary, based on an analysis of existing search interfaces and known behaviors for those looking for research data. The process around these discovery scenarios is to posit the thinking of the researcher/user seeking to find data, to identify needed classes and properties in the vocabulary, and then to render the search as it might be implemented.

\section{Link Publications to Datasets}

Publications, which describe ongoing research or its output based on research data, are typically held in bibliographical databases or information systems. By adding unique, persistent identifiers established in scholarly publishing to DDI-based metadata for datasets, these datasets become citable in research publications and thereby linkable and discoverable for users. And in addition the extension of research data with links to relevant publications is possible by adding citations and links. Such publications can directly describe study results in general or further information about specific details of a study, e.g., publications of methods or design of the study or about theories behind the study. Exposing and connecting additional material related to data described in DDI is already covered in DDI. In DDI-RDF, every element can be related to any foaf:Document using dcterms:relation. Researchers may also want to search for publications where specific questions are discussed.

\section{Discovering Studies Using Free Text Search in Study Descriptions}

The most natural way of searching for data is to formulate the information need by using free text terms and to match them against the most common metadata, like title, description, abstract, or unit of analysis. A researcher might search for relevant studies that have a particular title or keywords assigned to them in order to further explore the datasets. The definition of an analysis unit might help to directly determine which datasets the researcher wants to download afterwards. A typical query could be 'Find all studies with questions about commuting to work'.

\section{Searching for Studies by Publishing Agency}

Researchers are often aware of the organizations that disseminate the kind of data they want to use. This scenario shows how a researcher might wish to see the studies disseminated by a particular organization, so that the datasets that comprise them can be further explored and accessed. "Show me all the studies for the period 2000 to 2010 disseminated by the ESDS service of the UK Data Archive" is an example of a typical query. 


\section{Searching for Datasets by Accessibility}

This scenario describes how to retrieve datasets that fulfill particular access conditions. Many research datasets are not freely available, and access conditions may restrict some users from accessing some datasets. It is common to want to search only for those datasets that are either publicly available, or that have specific types of licensing/access conditions. Access conditions vary by country and institution. Users may be familiar with the specific licenses that apply in their own context. It is expected that the researcher looking for data might wish to see the datasets that meet specific access conditions or license terms. Here, a researcher is using a tool that will generate a SPARQL query that returns the titles of datasets that are, for example, publicly available under the Canadian Data Liberation Initiative Community policy. Optionally it would also be possible to provide links to the rights statement and the license.

There is a paper ${ }^{26}$ describing further possible use cases in detail. Researchers can search for studies by producer, contributor, coverage, universe (i.e., study population), and data source (e.g., study questionnaire). Social science researchers can search for datasets using variables, related questions, and classifications. Furthermore, one can search for reusable questions using related concepts, variables, universe, and coverage, or by text.

\section{RDF from Codebook and Lifecycle}

We have implemented a direct and a generic mapping between DDI-XML and DDI-RDF. DDI-Codebook and DDI-Lifecycle XML documents can be transformed automatically into an RDF representation corresponding to the ontology. The direct mappings are realized through XSLT stylesheets ${ }^{27}$. Bosch and Mathiak (2011) have developed a generic approach for designing domain ontologies. XML Schemas are converted to ontologies automatically using XSLT transformations, which are described in detail by Bosch and Mathiak (2012). After the transformation process, all the information located in the underlying XML Schemas of a specific domain is also stored in the generated ontologies. Domain ontologies can be inferred automatically out of the generated ontologies in a subsequent step (Bosch 2012). In this section, only the direct approach is described in detail.

The structure of DDI-Codebook differs substantially from DDILifecycle. DDI-C is designed to describe metadata for archival purposes, and the structure is very predictable and focused on describing variables with the option to add annotations for used question texts, etc. DDI-L on the other hand is designed to capture metadata from the early stages in the research process. A lot of the metadata can be described in modules, and references are used between, for example, questions and variables. DDI-L enables capturing and reuse of metadata through referencing.

The Disco vocabulary is developed with this in mind -- the discovery of studies, questions, and variables should be the same regardless of which version of DDI was used to document the study. DDI-L has more elements and is able to describe studies, variables, and questions in greater detail than DDI-C. However, the core metadata for the discovery purpose is available in both DDI-C and DDI-L. The transformation can be automated and standardized for both. That means that regardless of the input -- DDI-C or DDI-L -- the resulting RDF is the same. This enables an easy and equal search in RDF resulting from DDI-C and DDI-L. Also, interoperability between both is increased.

\section{Creating Triples from DDI XML via XSLT}

There is a huge ecosystem of tools exporting DDI-XML. This makes it possible to act on the output in a standardized way via XSLT. XSLT is implemented in a wide variety of environments and is a good method for making the transformation from DDI-XML to Disco. The flexibility of XSLT allows us to generate one conversion process for both DDI-C and DDI-L, which can be detected automatically inside the XSLT by paths and nodes of the input files. This corresponds to the goal to generate a consistent and equal Disco output independently of the DDI input.

The goal of making this implementation is to provide a simple way to start publishing DDI as RDF. XSLT is also easy to customize and extend so users can take the base and add output to other vocabularies if they have specialized requirements. It can also be adjusted if special requirements to the input are given. Keeping the XSLT as general as possible, we provide the basis for a broad reusability of the conversion process.

The implementation can also be used as a reference to show how elements in DDI-C and DDI-L map to Disco. The current version of the XSLT can be found at <https://github.com/linked-statistics/ DDI-RDF-tools>

\section{Future Work on the Mapping and DDI-RDF XSLT}

Currently, we have created two separate XSLT files for the conversion of DDI-C and DDI-L. According to the flexibility of XSLT we aim to merge them into one generic conversion XSLT that automatically detects which DDI input is given. Also, we plan on including parameters into the conversion process in order to select and define particular languages and URI prefixes.

Since the work on the conceptual model of Disco is currently not finished, the finalized mappings of DDI to Disco have to be included into the XSLT.

\section{Future Work on Integrated Use of Disco and Related RDF Vocabularies}

The description of the relationship of aggregated data to the original microdata by Disco, RDF Data Cube, and Prov will be further explored. Another focus will be how data portals can benefit of the combined use of Disco with DCAT, and the new RDF vocabulary on Physical Data Description (PHDD) ${ }^{28}$

\section{Conclusions}

In this paper, we introduced the DDI-RDF model, an approach for applying a non-RDF standard to the web of data. We developed an RDFS/OWL ontology for a basic subset of DDI to solve the most frequent and important problems associated with diverse use cases (especially for discovery purposes) and to open the DDI model to the Linked Open Data community. There are two implementations of mappings between DDI-XML and DDI-RDF: a direct mapping and a generic one, which can be applied within various contexts. The most important use cases associated with an ontology of the DDI data model are to find and link to publications related with particular data, to map terms to concepts of external thesauri, and to discover data and metadata that are interlinked with more than one study.

Diverse benefits are connected with the publication of DDI data and metadata in the form of RDF. Users of the DDI social science metadata standard can query multiple, distributed, and merged DDI instances using established Semantic Web technologies. 
Members of the DDI community can publish DDI data as well as metadata in the Linked Open Data cloud. Therefore, DDI instances can be processed by RDF tools without supporting and knowing the DDI-XML Schemas' data structures. After publishing public available structured data, DDI data and metadata can be connected with other data sources of multiple topical domains.

\section{Acknowledgements}

The work described in this paper was started at the first workshop on "Semantic Statistics for Social, Behavioral, and Economic Sciences: Leveraging the DDI Model for the Linked Data Web" 29 at Schloss Dagstuhl - Leibniz Center for Informatics, Germany in September 2011. This work was continued at three meetings: a follow-up working meeting in the course of the 3rd Annual

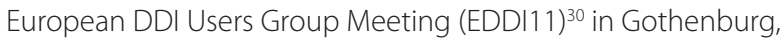
Sweden, in December 2011; a second workshop on "Semantic Statistics for Social, Behavioral, and Economic Sciences: Leveraging the DDI Model for the Linked Data Web" ${ }^{31}$ at Schloss Dagstuhl - Leibniz Center for Informatics, Germany in October 2012; and a follow-up working meeting at GESIS - Leibniz Institute for the Social Sciences in Mannheim, Germany, in February 2013. This work has been supported by contributions of the participants of the events mentioned above: Archana Bidargaddi (NSD - Norwegian Social Science Data Services), Thomas Bosch (GESIS - Leibniz Institute for the Social Sciences, Germany), Sarven Capadisli (Bern University of Applied Sciences, Switzerland), Franck Cotton (INSEE - Institut National de la Statistique et des Études Économiques, France), Richard Cyganiak (DERI, Digital Enterprise Research Institute, Ireland), Daniel Gillman (BLS - Bureau of Labor Statistics, USA), Arofan Gregory (ODaF - Open Data Foundation, USA and DDI Alliance Technical Implementation Committee), Rob Grim (Tilburg University, Netherlands), Marcel Hebing (SOEP - German SocioEconomic Panel Study), Larry Hoyle (University of Kansas, USA), Yves Jaques (FAO of the UN), Jannik Jensen (DDA - Danish Data Archive), Benedikt Kämpgen (Karlsruhe Institute of Technology, Germany), Stefan Kramer (CISER - Cornell Institute for Social and Economic Research, USA), Amber Leahey (Scholars Portal Project University of Toronto, Canada), Olof Olsson (SND - Swedish National Data Service), Heiko Paulheim (University of Mannheim, Germany), Abdul Rahim (Metadata Technologies Inc., USA), John Shepherdson (UK Data Archive), Dan Smith (Algenta Technologies Inc., USA), Humphrey Southall (Department of Geography, UK Portsmouth University), Wendy Thomas (MPC - Minnesota Population Center, USA and DDI Alliance Technical Implementation Committee), Johanna Vompras (University Bielefeld Library, Germany), Joachim Wackerow (GESIS - Leibniz Institute for the Social Sciences, Germany and DDI Alliance Technical Implementation Committee), Benjamin Zapilko (GESIS - Leibniz Institute for the Social Sciences, Germany), Matthäus Zloch (GESIS - Leibniz Institute for the Social Sciences, Germany).

\section{References}

Bosch, T., Cyganiak, R., Wackerow, J., and Zapilko, B. 2012. Leveraging the DDI Model for Linked Statistical Data in the Social, Behavioural, and Economic Sciences. International Conference on Dublin Core and Metadata Applications, 46-55.

Bosch, T. 2012. Reusing XML schemas' information as a foundation for designing domain ontologies. Proceedings of the 11th International Semantic Web Conference, Part II (Berlin, Heidelberg, 2012), 437-440. Bosch, T. and Mathiak, B. 2011. Generic Multilevel Approach Designing Domain Ontologies based on XML Schemas. Proceedings of the ISWC 2011 Workshop Ontologies Come of Age in the Semantic Web (OCAS) (Bonn, Germany, 2011), 1-12.
Bosch, T. and Mathiak, B. 2012. XSLT transformation generating OWL ontologies automatically based on XML Schemas. 6th International Conference for Internet Technology and Secured Transactions (ICITST) (Abu Dhabi, United Arab Emirates, 2012), 660 -667.

\section{Notes}

1. Thomas Bosch GESIS - Leibniz Institute for the Social Sciences, Mannheim, Germany E-Mail: thomas.bosch@gesis.org

2. Olof Olsson SND - Swedish National Data Service, Gothenburg, Sweden E-Mail: olof.olsson@snd.gu.se

3. Benjamin Zapilko GESIS - Leibniz Institute for the Social Sciences, Köln, Germany E-Mail: benjamin.zapilko@gesis.org

4. Arofan Gregory Open Data Foundation, Tucson, USA E-Mail: agregory@opendatafoundation.org

5. Joachim Wackerow GESIS - Leibniz Institute for the Social Sciences, Mannheim, Germany E-Mail: joachim.wackerow@gesis.org

6. http://sdmx.org/

7. http://www.ddialliance.org/ddi-at-work/projects

8. http://www.dataarchive.ac.uk/

9. http://thedata.org/

10. http://www.icpsr.umich.edu

11. http://www.abs.gov.au/

12. http://www.ihsn.org/adp

13. http://www.childinfo.org/mics3_surveys.html

14. http://data.worldbank.org/

15. http://www.theglobalfund.org/

16. http://www.ddialliance.org/what

17. http://www.loc.gov/standards/premis/

18. http://www.w3.org/2004/02/skos/

19. http://www.w3.org/TR/void/

20. http://www.w3.org/TR/prov-o/

21. http://www.w3.org/TR/vocab-dcat/

22. http://www.w3.org/TR/vocab-data-cube/

23. http://rdf-vocabulary.ddialliance.org/discovery

24. http://www.ddialliance.org/Specification/

25. http://www.w3.org/TR/vocab-data-cube/

26. http://www.ddialliance.org/resources/publications

27. https://github.com/linked-statistics/DDI-RDF-tools

28. http://rdf-vocabulary.ddialliance.org/phdd.html

29. http://www.dagstuhl.de/11372

30. http://www.iza.org/eddi1 1

31. http://www.dagstuhl.de/12422 